Research Article

\title{
The New Scramble for Faure Sequence Based on Irrational Numbers
}

\author{
Ali Mogharrabi O. $\left(\mathbb{D},{ }^{1}\right.$ Behrooz Fathi V. ${ }^{1},{ }^{2}$ M. H. Behzadi $\mathbb{D},{ }^{1}$ and R. Farnoosh $\mathbb{D D}^{3}$ \\ ${ }^{1}$ Department of Statistics, Science and Research Branch, Islamic Azad University, Tehran, Iran \\ ${ }^{2}$ Department of Statistics, University of Guilan, Rasht, Iran \\ ${ }^{3}$ Department of Mathematics, Iran University of Science and Technology, Tehran, Iran \\ Correspondence should be addressed to Behrooz Fathi V.; fathi@guilan.ac.ir
}

Received 24 December 2020; Revised 11 January 2021; Accepted 10 March 2021; Published 28 April 2021

Academic Editor: Leopoldo Greco

Copyright (c) 2021 S. A. Mogharrabi et al. This is an open access article distributed under the Creative Commons Attribution License, which permits unrestricted use, distribution, and reproduction in any medium, provided the original work is properly cited.

\begin{abstract}
This article intends to review quasirandom sequences, especially the Faure sequence to introduce a new version of scrambled of this sequence based on irrational numbers, as follows to prove the success of this version of the random number sequence generator and use it in future calculations. We introduce this scramble of the Faure sequence and show the performance of this sequence in employed numerical codes to obtain successful test integrals. Here, we define a scrambling matrix so that its elements are irrational numbers. In addition, a new form of radical inverse function has been defined, which by combining it with our new matrix, we will have a sequence that not only has a better close uniform distribution than the previous sequences but also is a more accurate and efficient tool in estimating test integrals.
\end{abstract}

\section{Introduction}

It is well known that Monte Carlo calculations are based on the generation of random numbers on interval $(0,1)$. Therefore, the generation of random numbers that have more uniformity on $(0,1)$ guarantees better approximations in these calculations. In recent years, some researchers have employed quasirandom sequences instead of random numbers to aim producing extra uniformity of the randomly generated numbers on $(0,1)$. Due to the breadth and complexity of some problems that are mostly unsolvable by classical mathematical methods or solving them with classical methods is associated with more time and computational cost, the stochastic solving of such cases with numerical methods and using the Monte Carlo method plays a key role. The quasirandom sequences are common in Monte Carlo calculations such as Faure, Halton, Niederreiter, and Sobol sequences, but due to the lack of complete success of these sequences in Monte Carlo computation, we use scrambled versions of them, all of which are designed to increase the uniformity of randomly (quasirandom) generated numbers on $(0,1)$, so that we can estimate the obtained solution to the desired unknown solution of the problem.

To resolve this problem, researchers are competing on the use of scrambled quasirandom generators based on their version of random number generation to provide more accurate results in Monte Carlo calculations.

Today, Monte Carlo and quasi-Monte Carlo methods are widely used to solve the computations of physical and mathematical problems. Quasi-Monte Carlo (QMC) methods play an alternative role for Monte Carlo methods. The advantage of these methods is that they use numbers to provide extera uniformity on unit hypercube. This feature has led to the use of these methods to estimate high-dimensional integrals (Niederreiter, 1992; Spanier and Maize, 1994) [1].

So far, several quasirandom sequences (or low discrepancy sequences) have been introduced for the QMC method. Such as the Faure sequence, the Halton sequence, and the Sobol sequence. Despite the fact that among these three sequences, the Faure sequence has better features in terms of discrepancy bound, but in practice, it is less used. Because, the convergence rate of this class of sequences is not so good 
512 points from Faure sequence in base 19

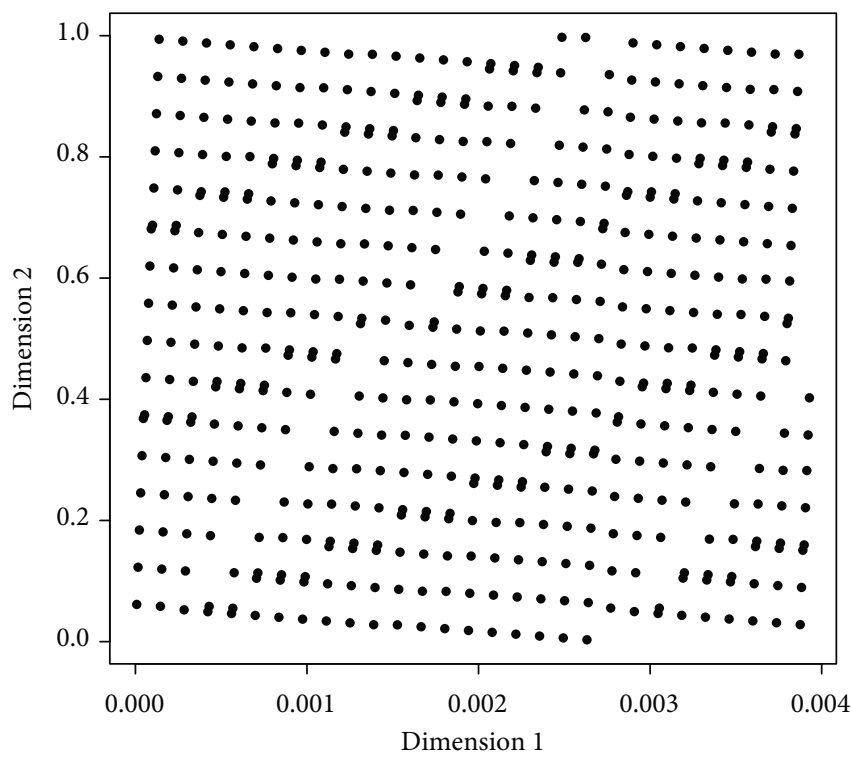

(a)

512 points from Faure sequence in base 71

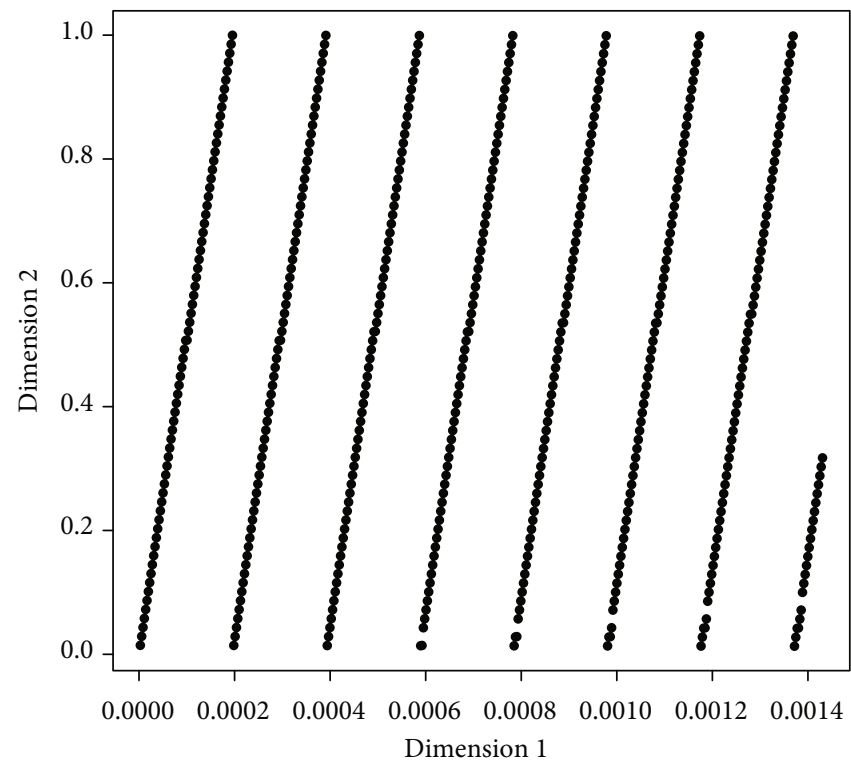

(c)

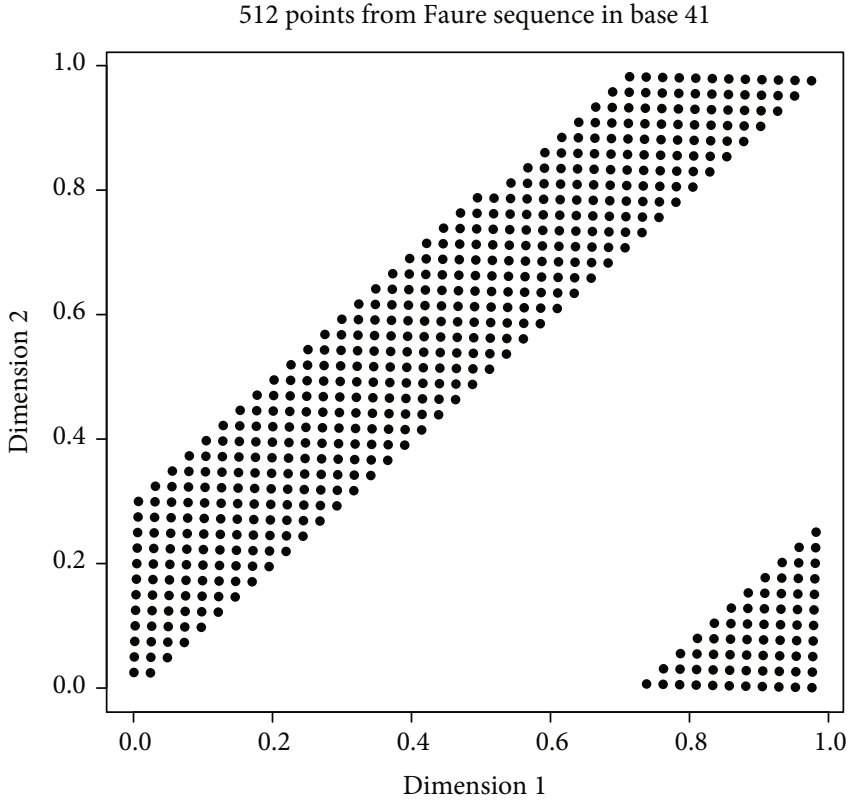

(b)

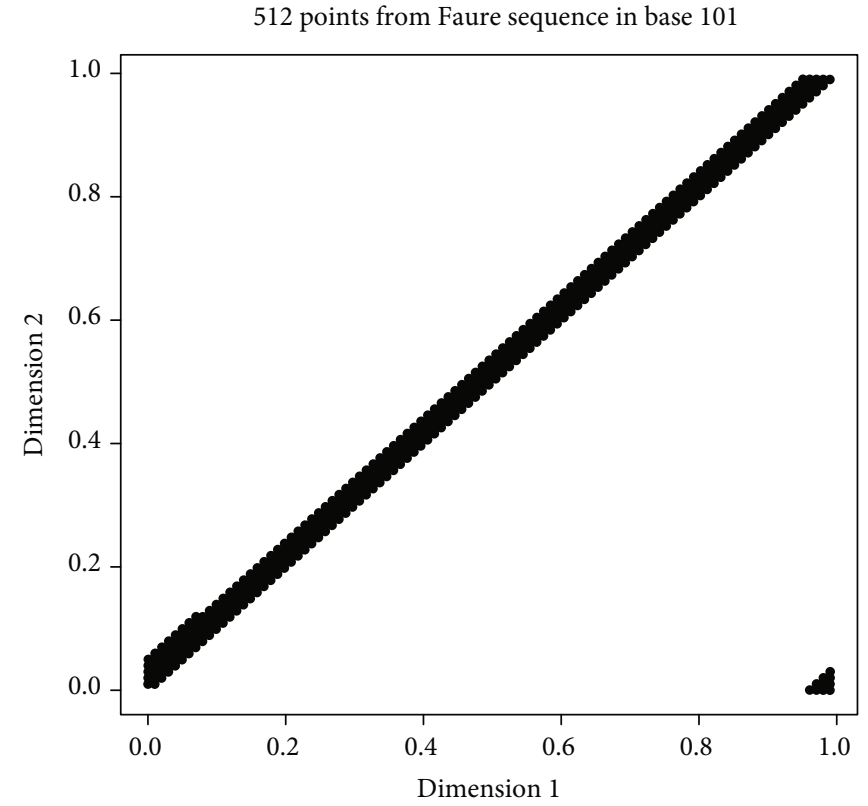

(d)

FIgURE 1: 512 points from the original Faure sequence in several bases.

compared with the other sequences [2]. In addition, due to the correlation between the different dimensions of the Faure sequence, the distribution of the sequence points is not very favorable, and we see poor two-dimensional projections (Figure 1). To overcome this problem, many scrambling methods have been proposed for the Faure sequence (see [3]). In almost all of these scrambles, there are attempts to define a new matrix by shifting numbers in the generating matrix or the placement of the elements, and there has been less talk about the properties of irrational numbers. The matrix that we introduce in this paper has been selected from several proposed matrices. Because it has good twodimensional projections and it is also at a very high level in terms of integral estimation.

In the next section, the structure of the original Faure sequence is given. We then briefly list the scramblers that have already been introduced in Section 3. In Section 4, we have brought our proposed matrix. Sections 5 and 6 give the evaluation criteria for the quality of the sequence generated by our proposed matrix and compare it with previous sequences, and in Section 7 , the conclusion is stated. 


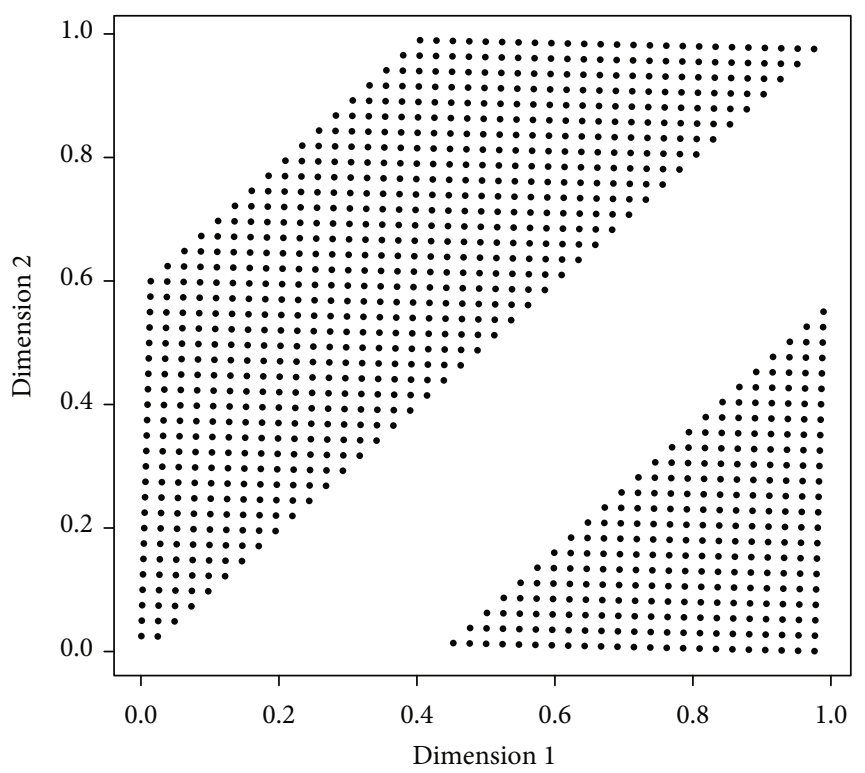

(a)

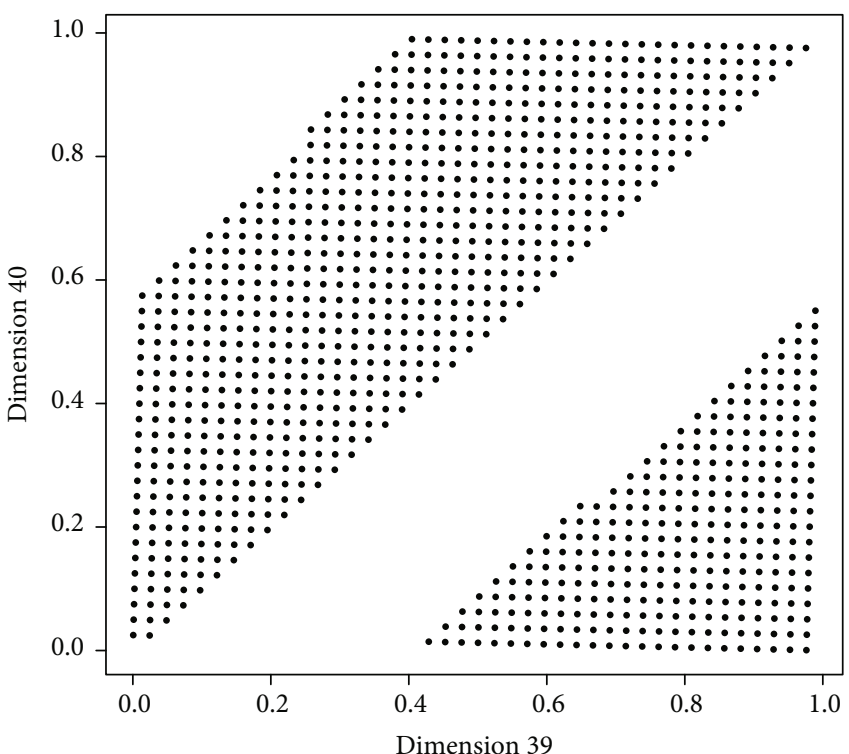

(b)

Figure 2: 1000 points from the original Faure sequence.

\section{The Faure Sequence}

Suppose $p \geq 2$ is a prime number, and suppose $N$ is the number of points we want to generate, and $\boldsymbol{n}=\left(a_{0}, a_{1}, \cdots, a_{m-1}\right)^{T}$ is a vector of integers whose components are from the expansion of the number $\boldsymbol{n}$ on the base $p$, where $0 \leq a_{j} \leq p$ and $m$ $=\left\lceil\log _{p} N\right\rceil$. We define the radical inverse function, $\phi_{p}(\boldsymbol{n})$, as

$$
\phi_{p}(\boldsymbol{n})=\frac{a_{0}}{p}+\frac{a_{1}}{p^{2}}+\cdots+\frac{a_{m-1}}{p^{m}} .
$$

For the Faure sequence, we define a different generator matrix for each dimension. If $\boldsymbol{P}$ be the Pascal matrix, then for a $s$-dimensional Faure sequence the generator matrix of the $j$ th dimension is $\boldsymbol{C}^{(j)}=\boldsymbol{P}^{j-1}, 1 \leq j \leq s$, where the member on the row $c$ and the column $r$ is defined as follows:

$$
\boldsymbol{P}^{j-1}=\left(\begin{array}{c}
c-1 \\
r-1
\end{array}\right)(j-1)^{c-r}(\bmod p), c \geq 1, r \geq 1 .
$$

Thus, let $x_{n}^{(j)}$ be represents the number $n$ in the dimension $j$ in the Faure sequence, then

$$
x_{n}^{(j)}=\phi_{p}\left(\boldsymbol{P}^{j-1} \boldsymbol{n}\right)
$$

and so the $\boldsymbol{s}$-dimensional Faure sequence is $\left(\phi_{p}\left(P^{0} n\right), \phi_{p}\right.$ $\left.\left(P^{1} n\right), \cdots, \phi_{p}\left(P^{s-1} n\right)\right)$.

\section{Scrambling the Faure Sequence}

Since the introduction of the Faure sequence, several methods were proposed to scramble it. In this section, we give an overview of some of such scrambles.
3.1. The Generalized Faure Sequence. Tezuka [2] proposed the generalized Faure sequence, GFaure, with the $j$ th dimension generator matrix $\boldsymbol{C}^{(j)}=\boldsymbol{A}^{(j)} \boldsymbol{P}^{j-1}$ and the $\boldsymbol{A}^{(j)}$ for $j=1,2$ $, \cdots, s$ are arbitrary nonsingular lower triangular matrices over $\mathbb{F}_{p}$. A special case for $A^{(j)}$ is that all members are one for all dimensions [4].

3.2. Random Linear (Digit) Scrambling. After reviewing different versions of the Owen's method, Matoušek introduced a scramble matrix and a transfer vector for various dimensions [5]. The sequences obtained by Matoušek have the following general form:

$$
\mathrm{x}_{n}=\left(\phi_{p}\left(\mathrm{~A}^{(1)} \boldsymbol{P}^{0} \boldsymbol{n}+\boldsymbol{g}_{1}\right), \phi_{p}\left(\mathrm{~A}^{(2)} \boldsymbol{P}^{1} \boldsymbol{n}+\boldsymbol{g}_{2}\right), \cdots, \phi_{p}\left(\mathrm{~A}^{(s)} \boldsymbol{P}^{s-1} \boldsymbol{n}+\boldsymbol{g}_{s}\right)\right)
$$

For the random linear scrambling, the matrices $A^{(j)}$ and the vectors $\boldsymbol{g}_{j}$ for $j=1, \cdots, s$ are of the form

$$
\boldsymbol{A}^{(j)}=\left(\begin{array}{cccc}
h_{1,1} & 0 & 0 & 0 \\
h_{2,1} & h_{2,2} & 0 & 0 \\
h_{3,1} & h_{3,2} & h_{3,3} & 0 \\
\vdots & \vdots & \vdots & \ddots
\end{array}\right), \boldsymbol{g}_{j}=\left(\begin{array}{c}
g_{1} \\
g_{2} \\
g_{3} \\
\vdots
\end{array}\right)
$$

where the $g_{j}$ 's and the $h_{i, j}$ with $i \geq j$ are chosen randomly and independently from $\left\{0,1, \cdots, b_{1}\right\}$, the $h_{j, j}$ 's are chosen randomly and independently from $\{1,2, \cdots, p-1\}$. 


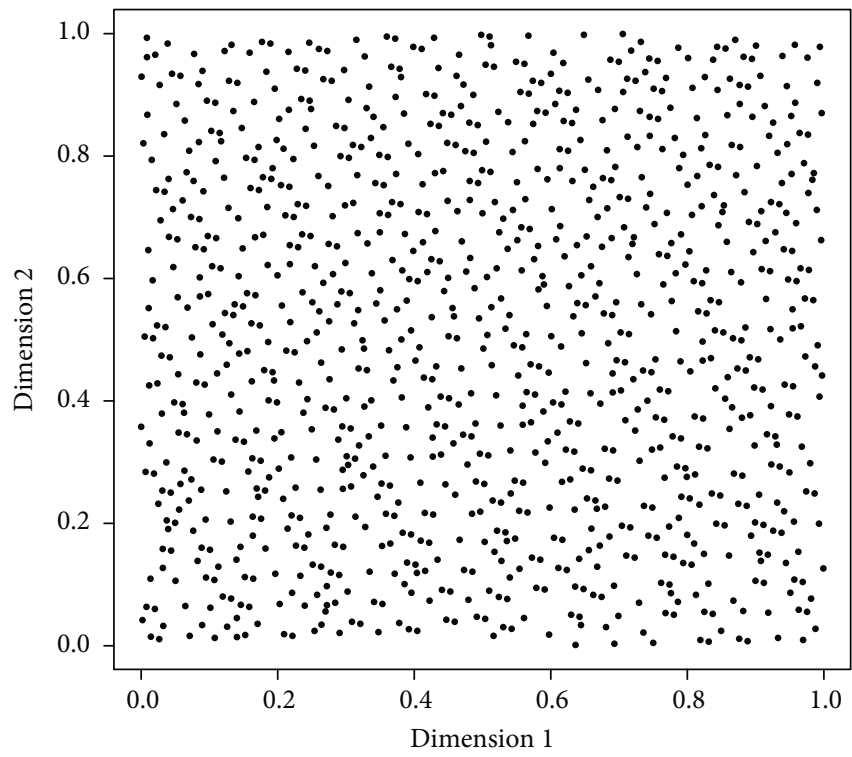

(a)

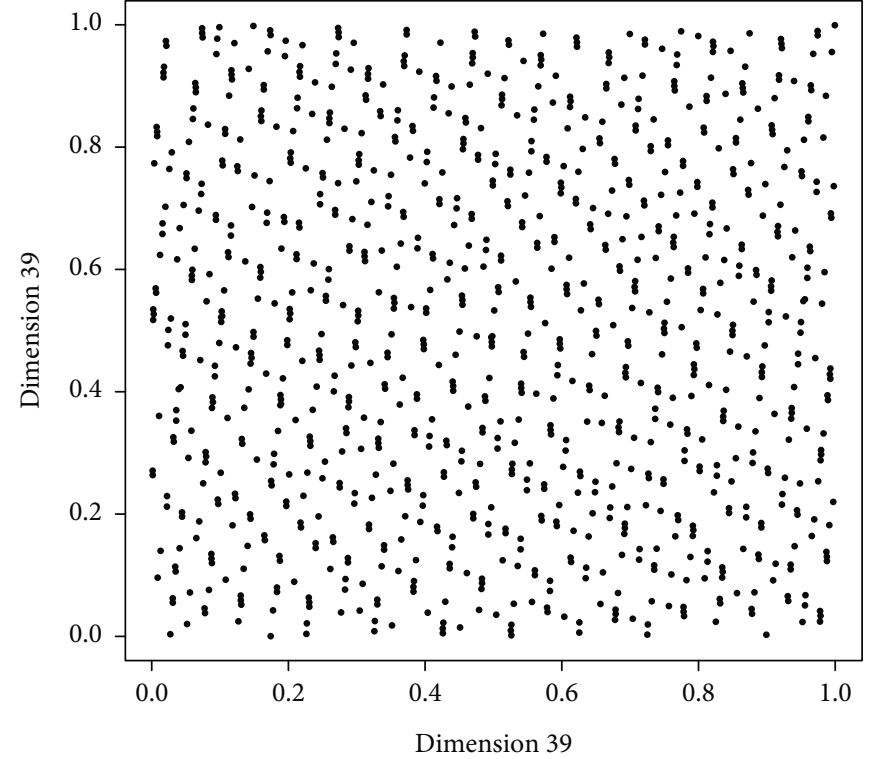

(b)

FIgURE 3: 1000 points from our scrambled Faure sequence.

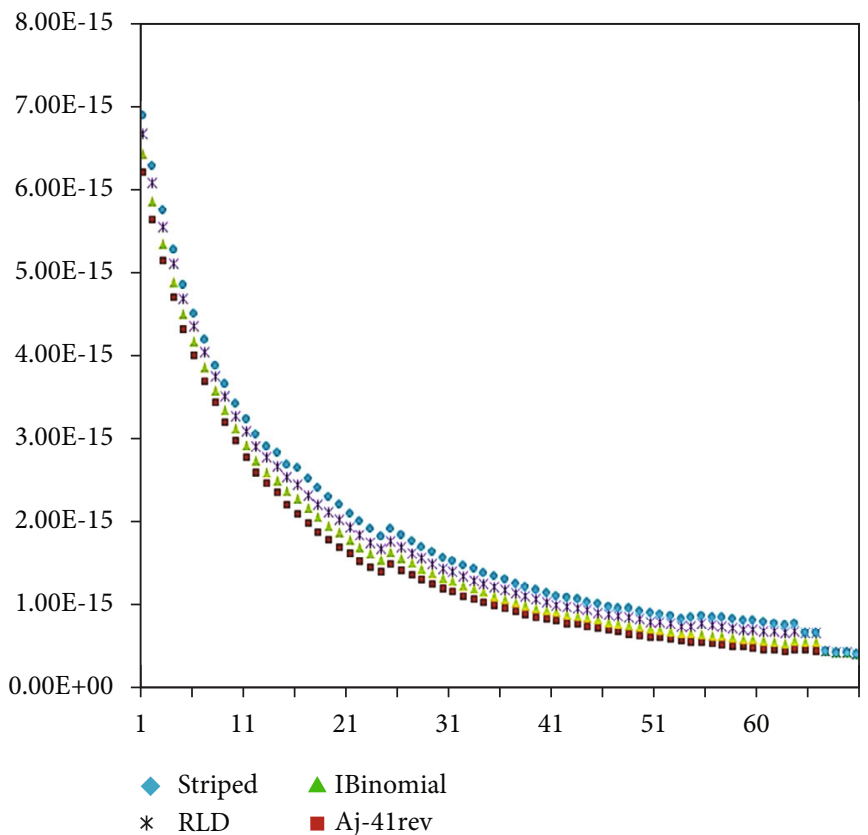

FIGURE 4: $L_{2}$-discrepancy for various randomized scramblings of a 40-dimensional Faure sequence.

Random linear digit method is the basis of other scrambles that will follow. Even the GFaure method is a subset of this method in which the members of the shift vectors are all zero.

3.3. I-Binomial Scrambling. A subset of the family of random linear scrambling methods is called left I-binomial scrambling [6]. Here, the $\boldsymbol{A}^{(j)}$ is defined as

$$
\boldsymbol{A}^{(j)}=\left(\begin{array}{ccccc}
h_{1} & 0 & 0 & 0 & 0 \\
h_{2} & h_{1} & 0 & 0 & 0 \\
h_{3} & h_{2} & h_{1} & 0 & 0 \\
h_{4} & h_{3} & h_{2} & h_{1} & 0 \\
\ddots & \ddots & \ddots & \ddots & \ddots \\
& & & &
\end{array}\right)
$$


Comparisons among Faure sequence $(s=10)$

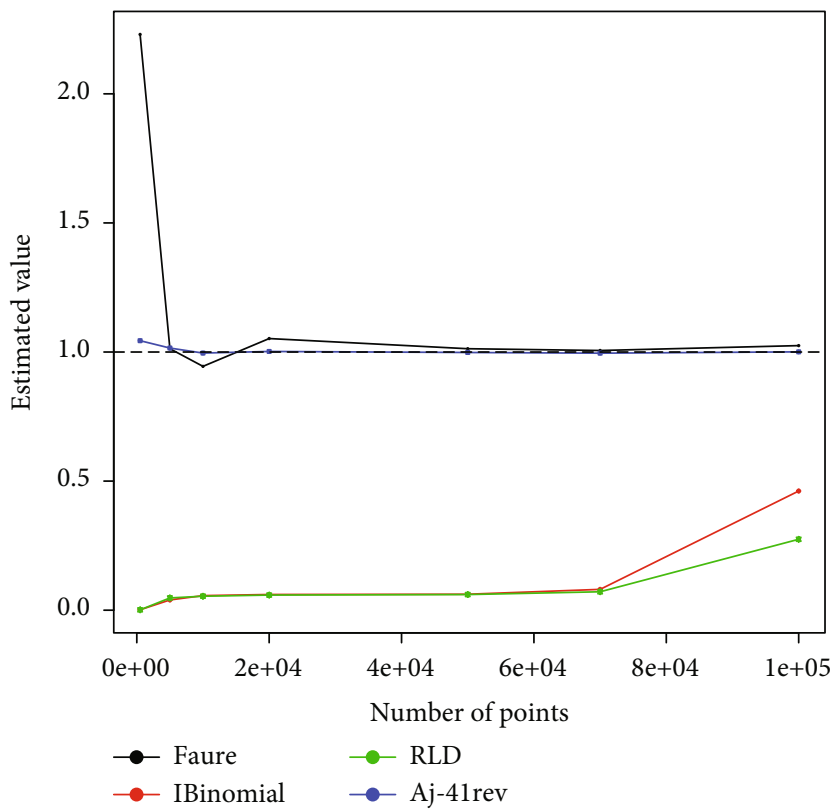

(a)

Comparisons among Faure sequence $(s=30)$

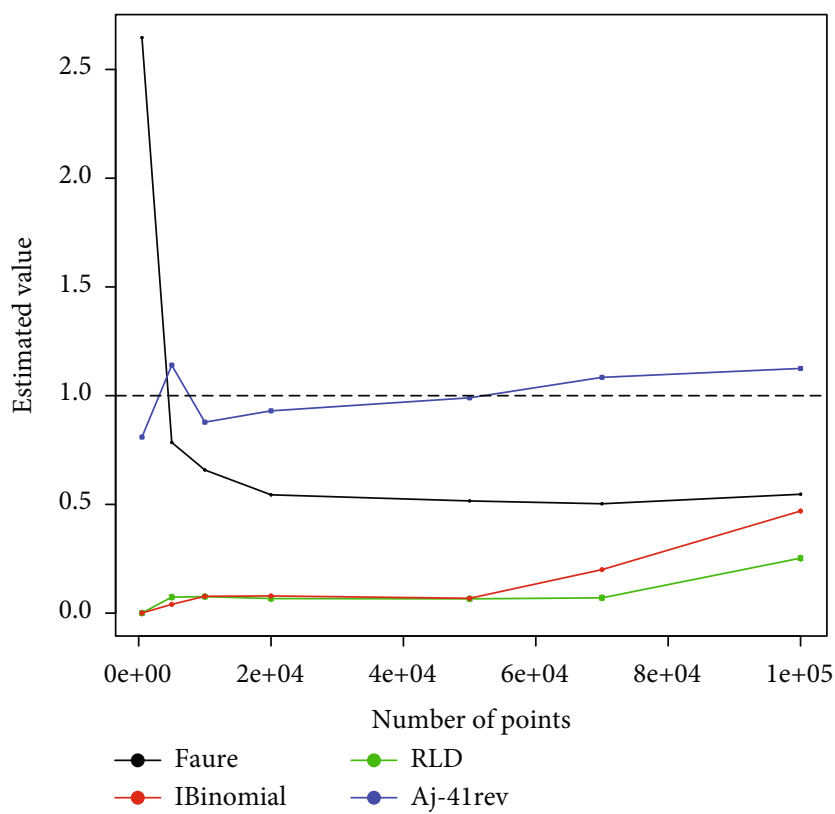

(c)
Comparisons among Faure sequence $(s=20)$

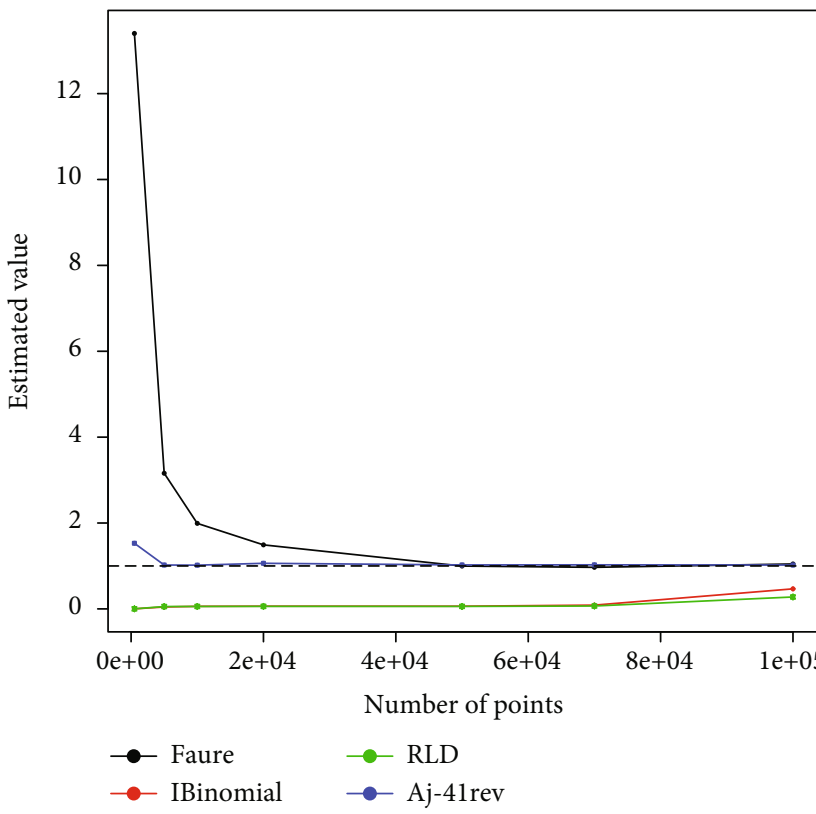

(b)

Comparisons among Faure sequence $(\mathrm{s}=40)$

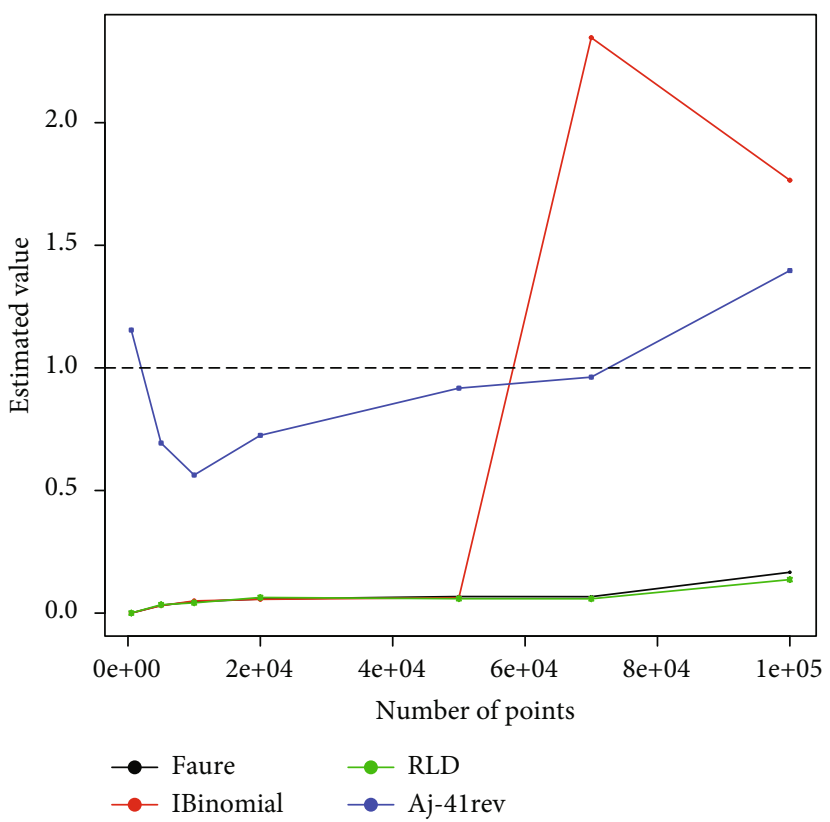

(d)

Figure 5: Estimates of the integral $I_{1}(f)$ by using various Faure sequences.

where $h_{1}$ is chosen randomly and independently from $\{1,2$ $, \cdots, p-1\}$ and also $h_{i}$ 's $(i>1)$ are chosen randomly and independently from $\{0,1, \cdots, p-1\}$.

3.4. Striped Matrix Scrambling. The scrambling matrix $\boldsymbol{A}^{(j)}$ for Striped Matrix Scrambling method, has the following form:

$$
\boldsymbol{A}^{(j)}=\left(\begin{array}{ccccc}
h_{1} & 0 & 0 & 0 & 0 \\
h_{1} & h_{2} & 0 & 0 & 0 \\
h_{1} & h_{2} & h_{3} & 0 & 0 \\
h_{1} & h_{2} & h_{3} & h_{4} & 0 \\
\vdots & \vdots & \vdots & \vdots & \vdots \\
& & & &
\end{array}\right),
$$


Comparisons among Faure sequence $(s=10)$

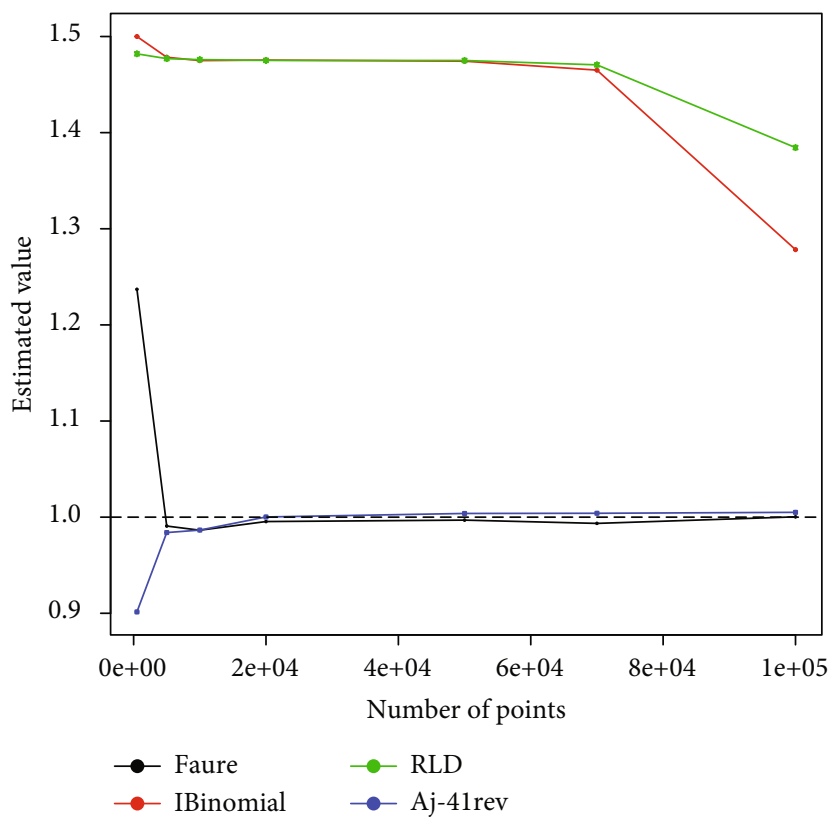

(a)

Comparisons among Faure sequence $(s=30)$

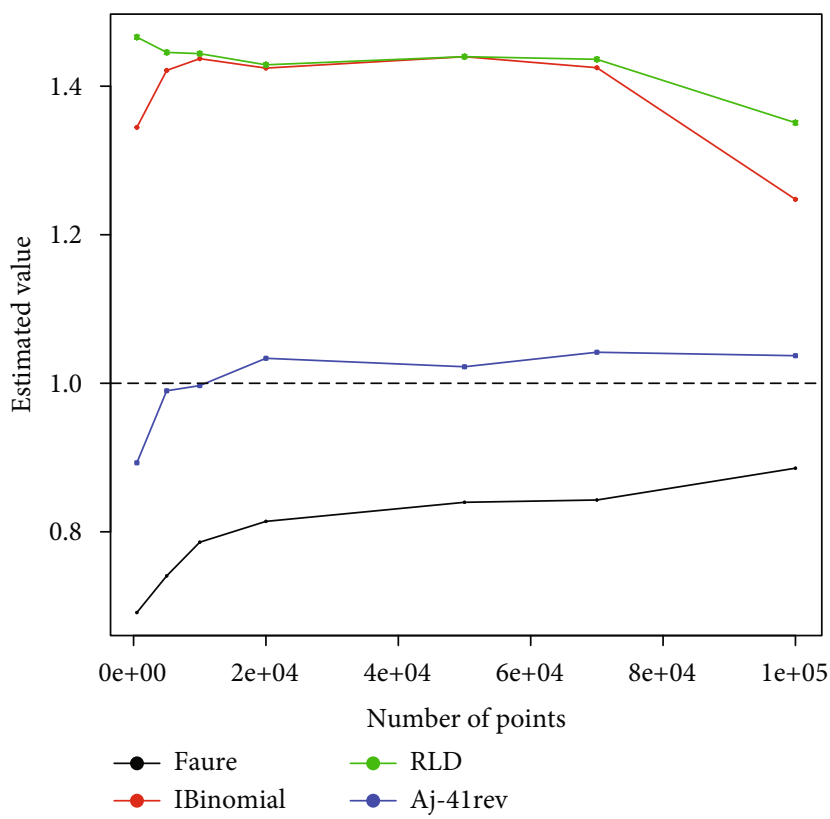

(c)

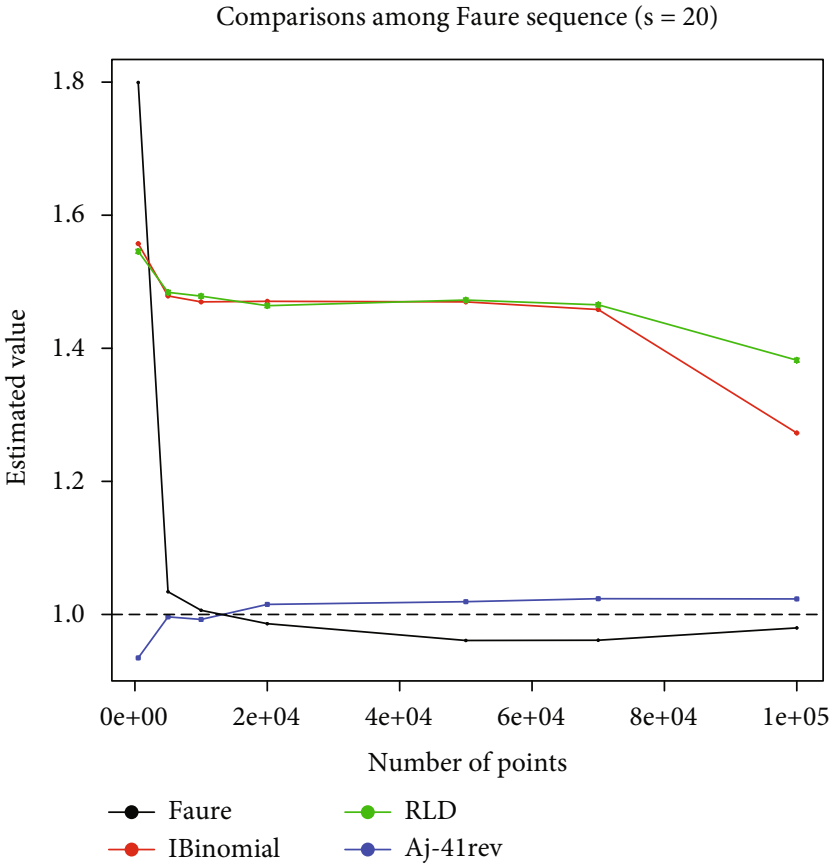

(b)

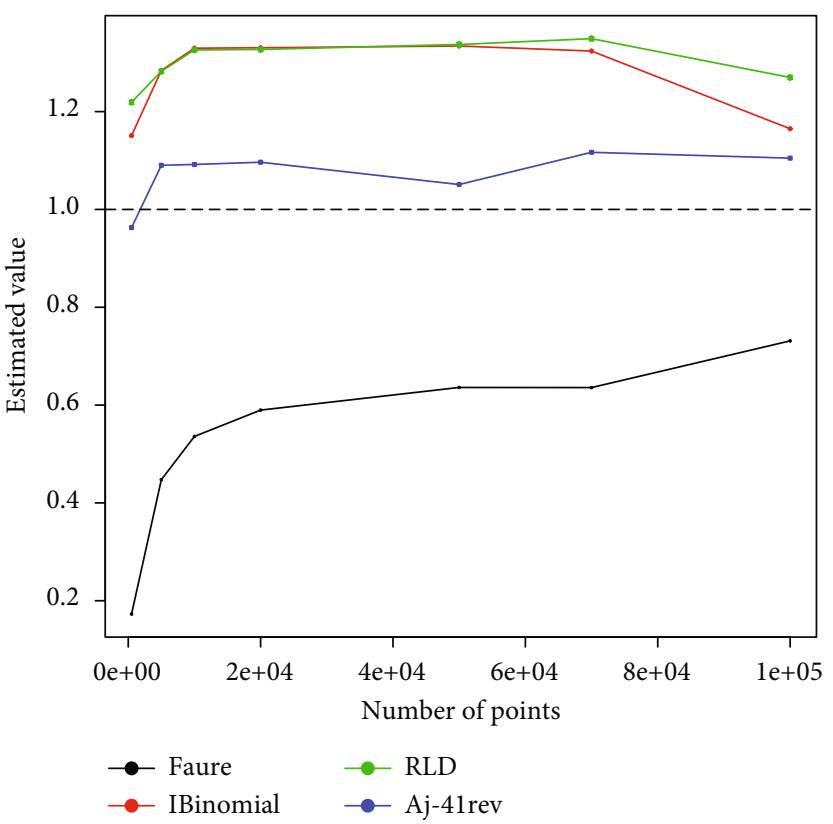

(d)

Figure 6: Estimates of the integral $I_{2}(f)$ with $a_{i}=1$ by using various Faure sequences.

where the $h_{i}$ 's are chosen randomly and independently from $\{1,2, \cdots, p-1\}$. The different types of this scramble are examined in [7], only for problems in the first dimension. Of course, it does not say what changes should be made to the matrix for higher dimensions.

3.5. Chi's Optimal Scramble. When we use the I-binomial method to scramble the Faure sequence, the value of (number) $h_{1}$ causes all the expansion digits of each number to be replaced (permuted). Now, if we leave out the first digit, the value of (number) $h_{2}$ causes all the remaining digits to be replaced (permuted).

So by cleverly selecting these two members, we can achieve better Faure sequences.

In [8], Chi has shown that the best choice for these two values can be obtained based on the primitive roots of the p. Finally, the Chi's optimal scramble matrix is as follows: 


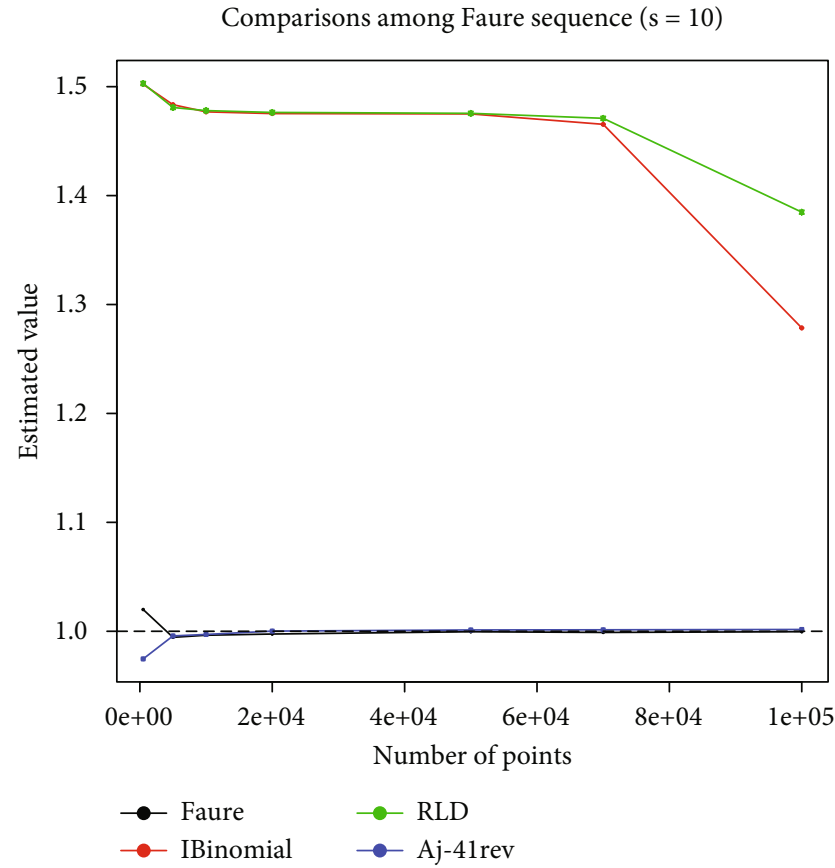

(a)

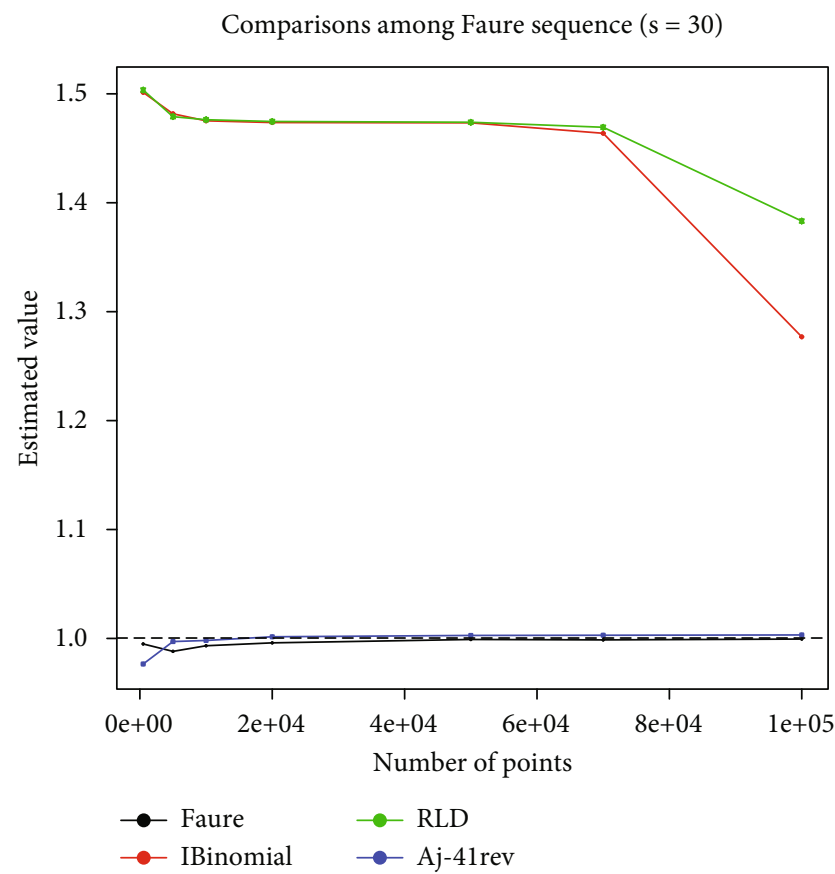

(c)

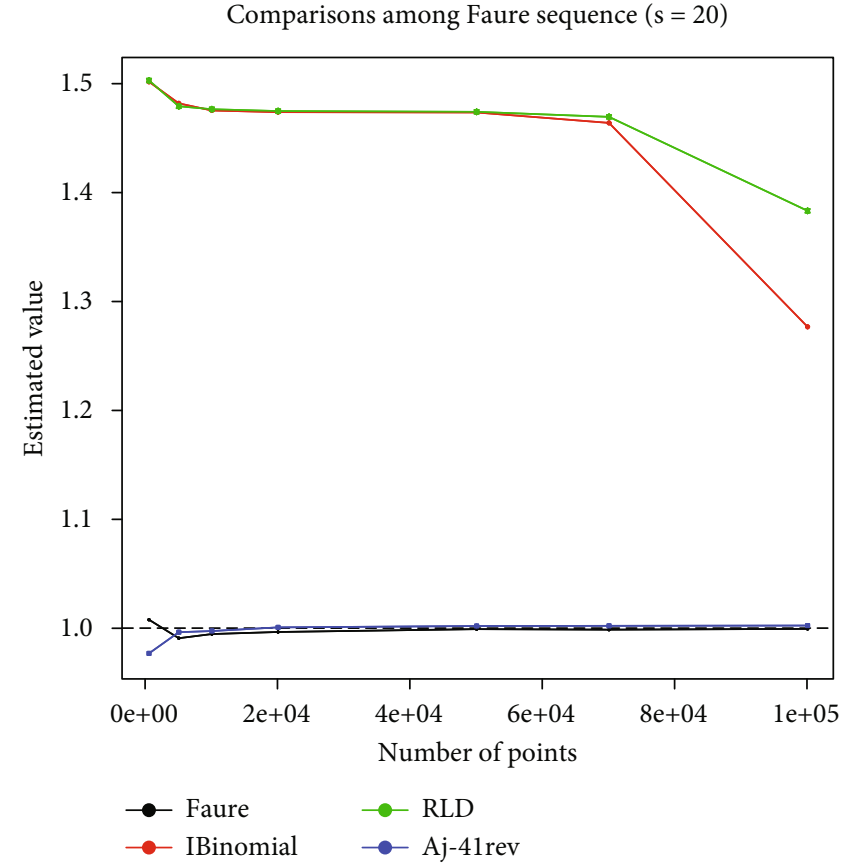

(b)

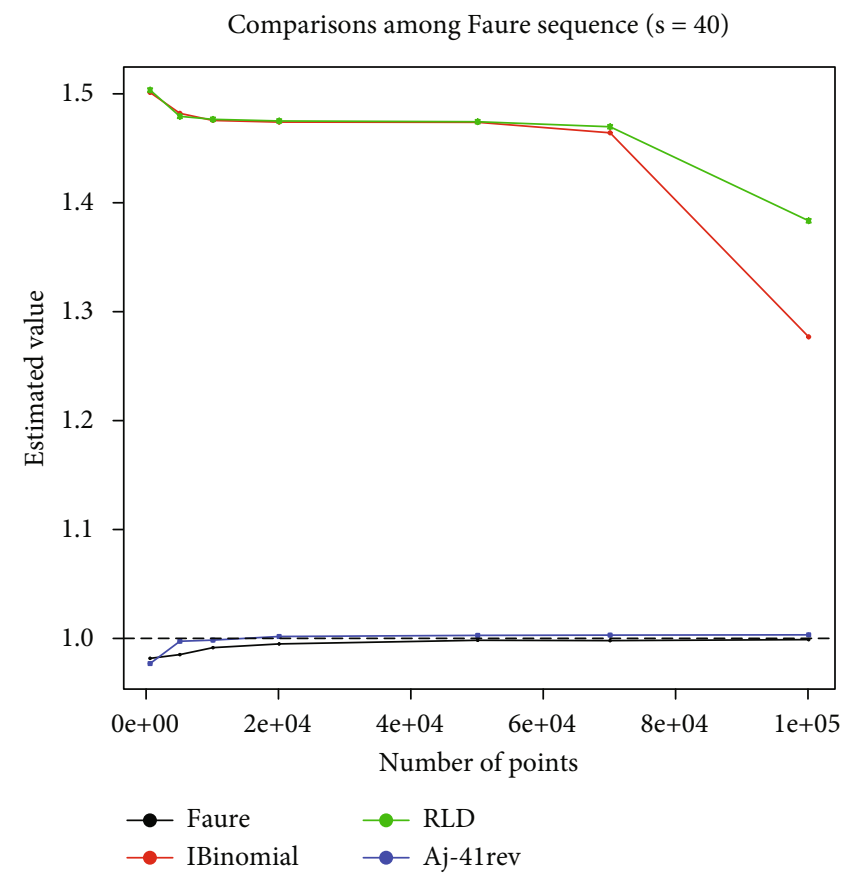

(d)

FIGURE 7: Estimates of the integral $I_{2}(f)$ with $a_{i}=i$ by using various Faure sequences. 


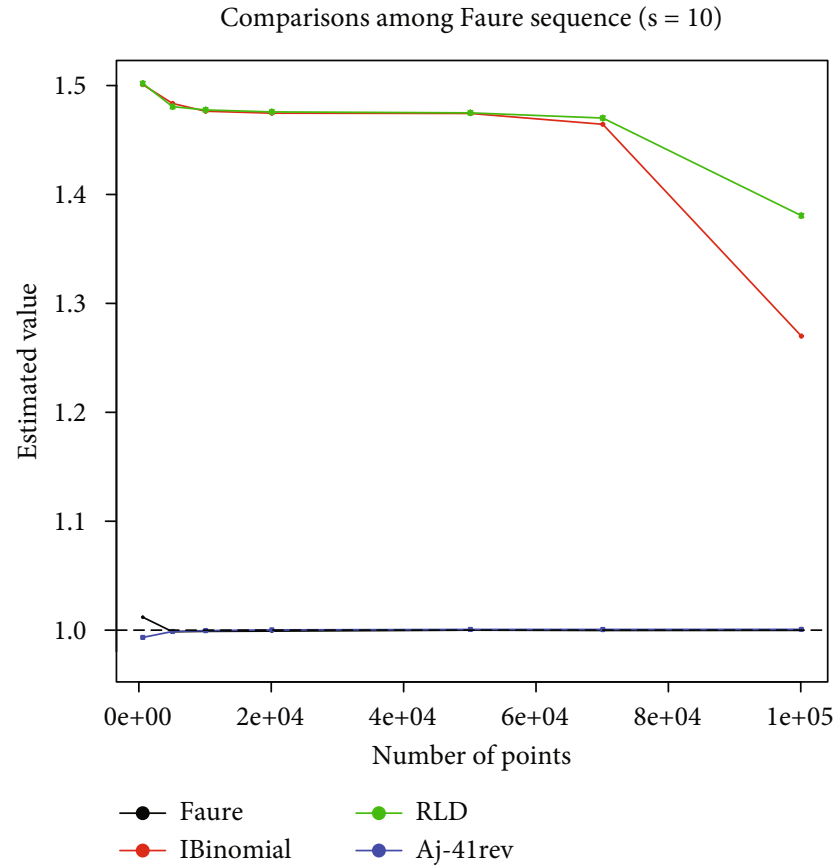

(a)

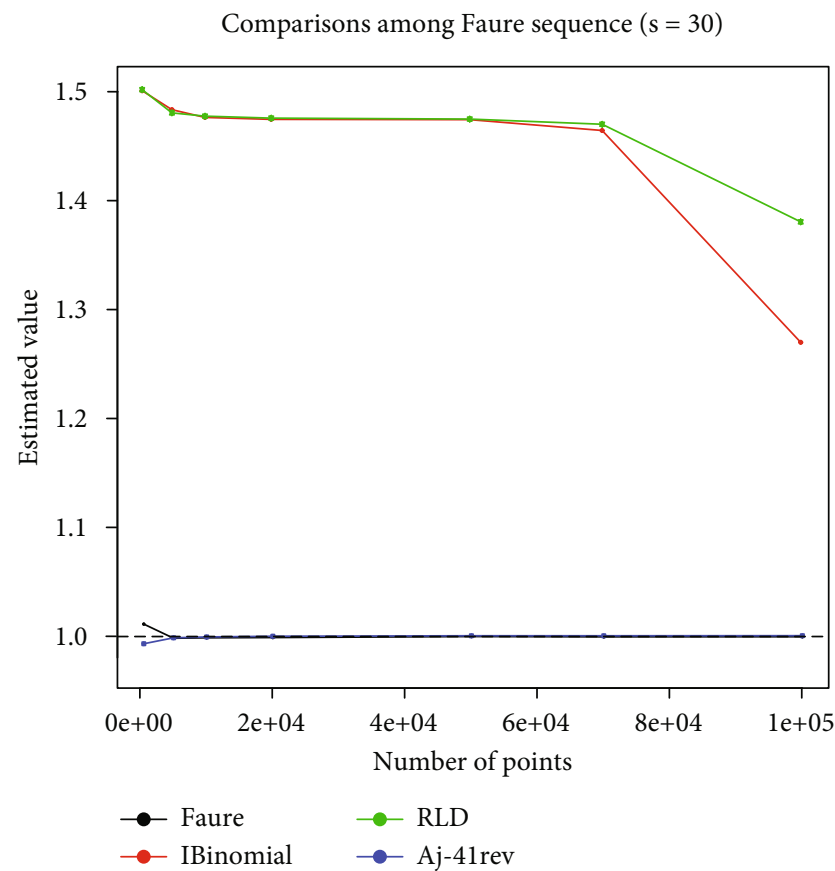

(c)

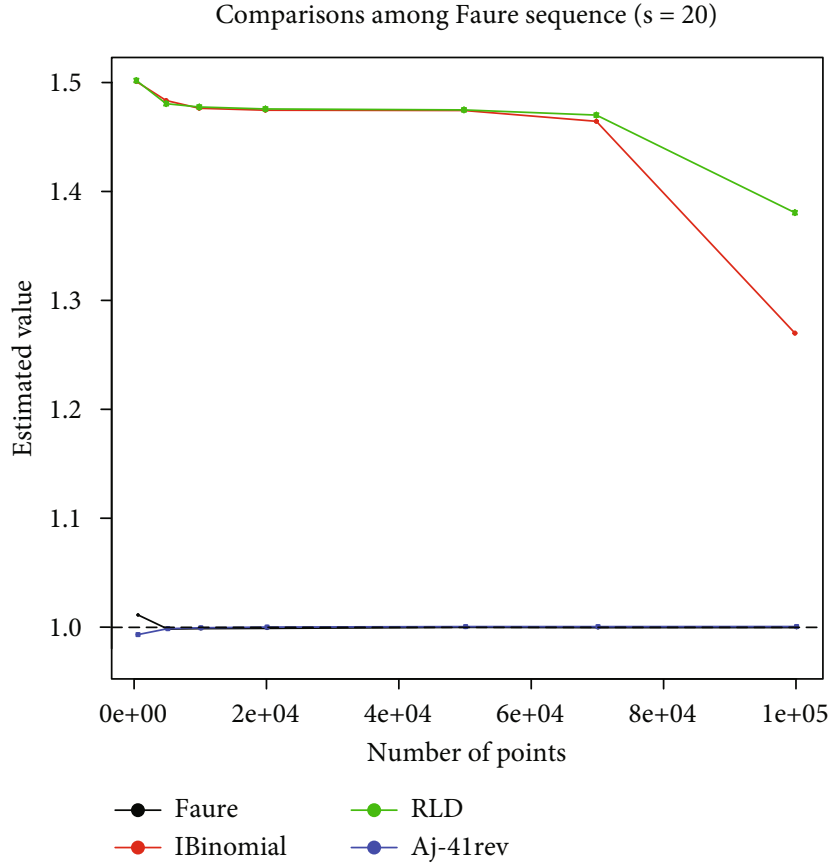

(b)

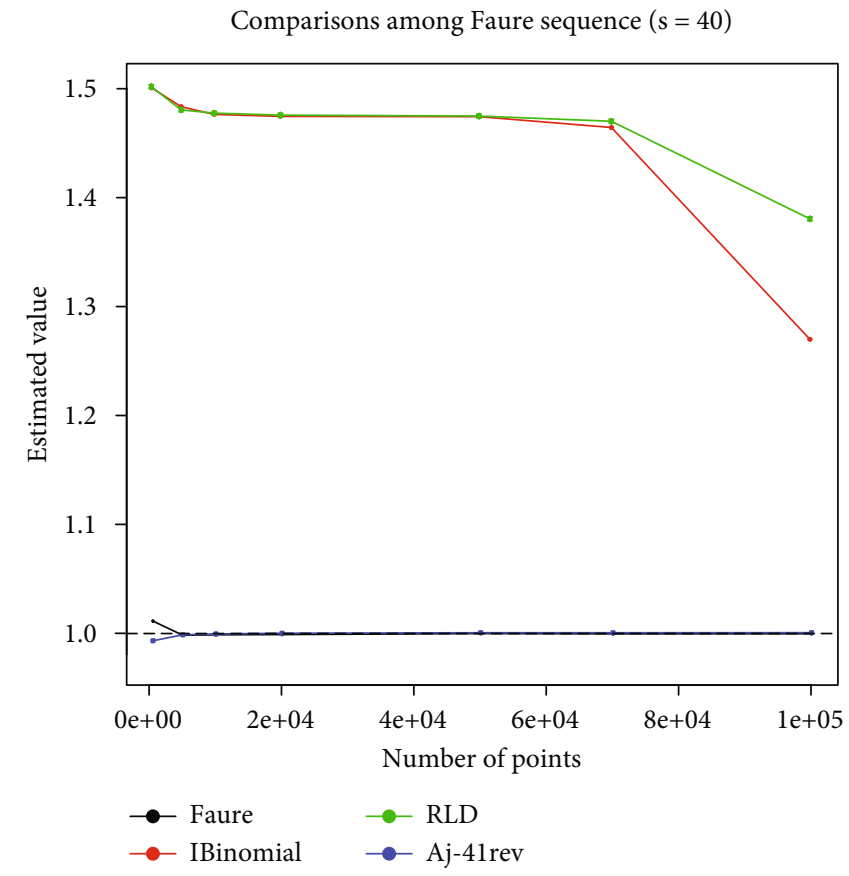

(d)

Figure 8: Estimates of the integral $I_{2}(f)$ with $a_{i}=i^{2}$ by using various Faure sequences. 


$$
\boldsymbol{A}^{(j)}=\left(\begin{array}{cccc}
h_{1}^{j-1} & 0 & 0 & 0 \\
h_{2} & h_{1}^{j-1} & 0 & 0 \\
0 & h_{2} & h_{1}^{j-1} & 0 \\
0 & 0 & h_{2} & h_{1}^{j-1} \\
\vdots & \vdots & \vdots & \ddots
\end{array}\right),
$$

3.6. Inverse Scrambling. Fathi and Eskandari [9] adapted Chi's optimal matrix and introduced two kind of matrix $\boldsymbol{A}^{(j)}$ as follows:

$$
\boldsymbol{A}_{1}^{(j)}=\left(\begin{array}{cccc}
d^{j-1} & 0 & 0 & 0 \\
0 & d^{j-1} & 0 & 0 \\
0 & 0 & d^{j-1} & 0 \\
\vdots & \vdots & \vdots & \ddots
\end{array}\right), \quad \boldsymbol{A}_{2}^{(j)}=\left(\begin{array}{cccc}
d^{j-1} & 0 & 0 & 0 \\
0 & d^{j} & 0 & 0 \\
0 & 0 & d^{j+1} & 0 \\
\vdots & \vdots & \vdots & \ddots
\end{array}\right),
$$

where $d$ is chosen from $\{1,2, \cdots, p-1\}$ for $j=1, \cdots, s$.

Based on nonlinear congruential method, they proposed another scrambling method for the Faure sequence for which the $j$ th coordinate of the $n$th point has the general form

$$
x_{n}^{(j)}=\phi_{p}\left(\Phi^{-1}\left(\boldsymbol{A}^{(j)} \Psi\left(\boldsymbol{P}^{j-1} \boldsymbol{n}\right)+\boldsymbol{g}_{j}\right)\right),
$$

where $\Phi(\boldsymbol{x})$ and $\Psi(\boldsymbol{x})$ are bijections that map a digit vector $\boldsymbol{x}$ to another digit vector.

\section{Scrambling Matrix with Irrational Members}

In this section, corresponding to the method of random linear digits, we introduce a scrambling matrix that its members are a function of square root of base $p$ (that is, they are irrational numbers).

After testing many functions, we found the following function that has the most performance:

$$
\boldsymbol{A}^{(j)}=\left(\begin{array}{cccc}
\sqrt{p}-1 & 0 & 0 & 0 \\
\sqrt{p}-1 & \sqrt{p}-1 & 0 & 0 \\
\sqrt{p}-1 & \sqrt{p}-1 & \sqrt{p}-1 & 0 \\
\sqrt{p}-1 & \sqrt{p}-1 & \sqrt{p}-1 & \sqrt{p}-1
\end{array}\right) .
$$

In (1), we introduced the common form of radical inverse function $\phi$ for base $p$. Now, we define a new form of this function as follows:

$$
\phi_{p}^{\prime}(\boldsymbol{n})=\frac{a_{0}}{p^{m}}+\frac{a_{1}}{p^{m-1}}+\cdots+\frac{a_{m-1}}{p} .
$$

TABLE 1: Estimates of $I_{1}(f)$ by using Faure sequences.

\begin{tabular}{lcccccc}
\hline Generator & $N$ & $s=5$ & $s=10$ & $s=20$ & $s=30$ & $s=40$ \\
\hline Faure & 500 & 1.2175 & 2.2301 & 13.402 & 2.6464 & 0.0001 \\
IB & 500 & 0.0017 & 0.0016 & 0.0014 & 0.0006 & 0.0002 \\
RLD & 500 & 0.0013 & 0.0013 & 0.0011 & 0.0006 & 0.0001 \\
Aj-41rev & 500 & 1.0392 & 1.0439 & 1.5286 & 0.8095 & 1.1542 \\
Faure & 5000 & 0.9803 & 1.0128 & 3.1585 & 0.7848 & 0.0314 \\
IB & 5000 & 0.0340 & 0.0397 & 0.0443 & 0.0402 & 0.0306 \\
RLD & 5000 & 0.0469 & 0.0475 & 0.0548 & 0.0737 & 0.0341 \\
Aj-41rev & 5000 & 1.0015 & 1.0154 & 1.0219 & 1.1401 & 0.6935 \\
Faure & 10000 & 0.9584 & 0.9445 & 1.9919 & 0.6583 & 0.0464 \\
IB & 10000 & 0.0569 & 0.0564 & 0.0593 & 0.0774 & 0.0496 \\
RLD & 10000 & 0.0539 & 0.0541 & 0.0566 & 0.0758 & 0.0418 \\
Aj-41rev & 10000 & 0.9992 & 0.9959 & 1.0182 & 0.8780 & 0.5630 \\
Faure & 20000 & 0.9943 & 1.0522 & 1.4909 & 0.5440 & 0.0575 \\
IB & 20000 & 0.0610 & 0.0607 & 0.0628 & 0.0791 & 0.0561 \\
RLD & 20000 & 0.0583 & 0.0585 & 0.0592 & 0.0670 & 0.0637 \\
Aj-41rev & 20000 & 0.9999 & 1.0021 & 1.0614 & 0.9301 & 0.7249 \\
Faure & 50000 & 0.9965 & 1.0127 & 0.9972 & 0.5159 & 0.0674 \\
IB & 50000 & 0.0617 & 0.0618 & 0.0621 & 0.0683 & 0.0628 \\
RLD & 50000 & 0.0604 & 0.0605 & 0.0582 & 0.0657 & 0.0587 \\
Aj-41rev & 50000 & 0.9984 & 0.9983 & 1.0241 & 0.9901 & 0.9173 \\
Faure & 70000 & 0.9964 & 1.0058 & 0.9680 & 0.5029 & 0.0669 \\
IB & 70000 & 0.0809 & 0.0806 & 0.0877 & 0.2000 & 2.3467 \\
RLD & 70000 & 0.0711 & 0.0713 & 0.0671 & 0.0702 & 0.0583 \\
Aj-41rev & 70000 & 0.9985 & 0.9954 & 1.0260 & 1.0841 & 0.9623 \\
Faure & 100000 & 1.0008 & 1.0248 & 1.0459 & 0.5466 & 0.1665 \\
IB & 100000 & 0.4610 & 0.4616 & 0.4667 & 0.4694 & 1.7654 \\
RLD & 100000 & 0.2742 & 0.2746 & 0.2772 & 0.2529 & 0.1366 \\
Aj-41rev & 100000 & 0.9979 & 1.0007 & 1.0215 & 1.1251 & 1.3969 \\
\hline & & & & & &
\end{tabular}

We call this as reverse radical inverse function, and we denote the sequences that are made in this way, with the suffix "rev." Therefore, by combining the matrix $\boldsymbol{A}^{(j)}$ and function $\phi^{\prime}$, the general form of number $n$ in dimension $j$ will be as follows:

$$
x_{n}^{(j)}=\phi_{p}^{\prime}\left(\boldsymbol{A}^{(j)} \boldsymbol{P}^{j-1} \boldsymbol{n}\right) .
$$

So, for example, we denote the 40-dimensional Faure sequence generated on the base 41 by the scrambled matrix $\boldsymbol{A}^{(j)}$ and the function $\phi^{\prime}$ with $\boldsymbol{A}^{(j)} .41 \mathrm{rev}$.

In the following sections, we have examined (studied) the quality of this sequence along with its performance compared to other sequences.

\section{Investigation of the Uniformity of Generated Sequences}

5.1. Two-Dimensional Projections. The first step in evaluating the performance of a sequence is to see how the points in the 2D projections are distributed. From Figure 2, in these designs, the Faure sequence points are located within parallel 
TABLE 2: Estimates of $I_{2}(f)$ with $a_{i}=1$ by using Faure sequences.

\begin{tabular}{lcccccc}
\hline Generator & $N$ & $s=5$ & $s=10$ & $s=20$ & $s=30$ & $s=40$ \\
\hline Faure & 500 & 1.0759 & 1.2370 & 1.7994 & 0.6913 & 0.1727 \\
IB & 500 & 1.5015 & 1.5000 & 1.5572 & 1.3445 & 1.1510 \\
RLD & 500 & 1.5123 & 1.4820 & 1.5456 & 1.4661 & 1.2191 \\
Aj-41rev & 500 & 0.9879 & 0.9014 & 0.9347 & 0.8930 & 0.9629 \\
Faure & 5000 & 0.9963 & 0.9908 & 1.0342 & 0.7406 & 0.4475 \\
IB & 5000 & 1.4836 & 1.4785 & 1.4787 & 1.4212 & 1.2841 \\
RLD & 5000 & 1.4807 & 1.4770 & 1.4840 & 1.4455 & 1.2824 \\
Aj-41rev & 5000 & 0.9976 & 0.9840 & 0.9964 & 0.9898 & 1.0902 \\
Faure & 10000 & 0.9935 & 0.9864 & 1.0062 & 0.7860 & 0.5359 \\
IB & 10000 & 1.4769 & 1.4750 & 1.4697 & 1.4370 & 1.3296 \\
RLD & 10000 & 1.4779 & 1.4759 & 1.4784 & 1.4438 & 1.3261 \\
Aj-41rev & 10000 & 0.9990 & 0.9865 & 0.9925 & 0.9968 & 1.0920 \\
Faure & 20000 & 0.9945 & 0.9954 & 0.9861 & 0.8140 & 0.5897 \\
IB & 20000 & 1.4753 & 1.4755 & 1.4706 & 1.4244 & 1.3306 \\
RLD & 20000 & 1.4763 & 1.4752 & 1.4640 & 1.4288 & 1.3271 \\
Aj-41rev & 20000 & 1.0000 & 1.0002 & 1.0151 & 1.0336 & 1.0965 \\
Faure & 50000 & 0.9992 & 0.9969 & 0.9609 & 0.8397 & 0.6360 \\
IB & 50000 & 1.4750 & 1.4744 & 1.4697 & 1.4397 & 1.3342 \\
RLD & 50000 & 1.4755 & 1.4751 & 1.4725 & 1.4398 & 1.3372 \\
Aj-41rev & 50000 & 1.0015 & 1.0039 & 1.0192 & 1.0222 & 1.0509 \\
Faure & 70000 & 0.9983 & 0.9935 & 0.9613 & 0.8428 & 0.6458 \\
IB & 70000 & 1.4654 & 1.4650 & 1.4582 & 1.4249 & 1.3238 \\
RLD & 70000 & 1.4710 & 1.4705 & 1.4653 & 1.4362 & 1.3491 \\
Aj-41rev & 70000 & 1.0020 & 1.0040 & 1.0237 & 1.0418 & 1.1168 \\
Faure & 100000 & 0.9997 & 1.0003 & 0.9798 & 0.8856 & 0.7312 \\
IB & 100000 & 1.2785 & 1.2783 & 1.2727 & 1.2476 & 1.1649 \\
RLD & 100000 & 1.3848 & 1.3844 & 1.3821 & 1.3508 & 1.2698 \\
Aj-41rev & 100000 & 1.0027 & 1.0050 & 1.0233 & 1.0371 & 1.1048 \\
\hline & & & & & &
\end{tabular}

lines, which shows that there is a linear correlation between points in successive dimensions. Also in these designs, we see a lot of empty spaces. Therefore, the distribution of good points has not been done. In Figure 3, we draw the same designs using the by $\boldsymbol{A}^{(j)} .41 \mathrm{rev}$.

These figures show that two-dimensional projections of $\boldsymbol{A}^{(j)}$.41 rev sequence are better than two-dimensional projections of the original Faure sequence.

5.2. Discrepancy. One way to measure the quality of a sequence is to calculate its discrepancy [10]. Warnock shows that

$$
\left(T_{N}\right)^{2}=\frac{1}{N^{2}} \sum_{k=1}^{N} \sum_{m=1}^{N} \prod_{i=1}^{s}\left(1-\max \left(x_{k}^{(i)}, x_{m}^{(i)}\right)\right)-\frac{2^{1-s}}{N} \sum_{k=1}^{N} \prod_{i=1}^{s}\left(1-x_{k}^{(i)^{2}}\right)+3^{-s} .
$$

where $x_{k}^{(i)}$ is the $i$ th component of the point $\boldsymbol{x}_{k}$.

Figure 4 compares $T_{N}$ between the original Faure sequence and the some scrambled Faure sequences that introduced in Section 3. From Figure 4, we see that the dis-
TABLE 3: Estimates of $I_{2}(f)$ with $a_{i}=i$ by using Faure sequences.

\begin{tabular}{lcccccc}
\hline Generator & $N$ & $s=5$ & $s=10$ & $s=20$ & $s=30$ & $s=40$ \\
\hline Faure & 500 & 1.0243 & 1.0200 & 1.0077 & 0.9945 & 0.9817 \\
IB & 500 & 1.5010 & 1.5022 & 1.5031 & 1.5030 & 1.5023 \\
RLD & 500 & 1.5055 & 1.5029 & 1.5043 & 1.5051 & 1.5045 \\
Aj-41rev & 500 & 0.9951 & 0.9746 & 0.9768 & 0.9761 & 0.9770 \\
Faure & 5000 & 0.9972 & 0.9944 & 0.9908 & 0.9877 & 0.9851 \\
IB & 5000 & 1.4837 & 1.4835 & 1.4833 & 1.4833 & 1.4832 \\
RLD & 5000 & 1.4810 & 1.4809 & 1.4807 & 1.4806 & 1.4804 \\
Aj-41rev & 5000 & 0.9990 & 0.9957 & 0.9963 & 0.9968 & 0.9974 \\
Faure & 10000 & 0.9977 & 0.9964 & 0.9946 & 0.9929 & 0.9915 \\
IB & 10000 & 1.4770 & 1.4769 & 1.4768 & 1.4768 & 1.4766 \\
RLD & 10000 & 1.4781 & 1.4780 & 1.4779 & 1.4778 & 1.4777 \\
Aj-41rev & 10000 & 0.9998 & 0.9971 & 0.9973 & 0.9977 & 0.9984 \\
Faure & 20000 & 0.9979 & 0.9977 & 0.9965 & 0.9956 & 0.9949 \\
IB & 20000 & 1.4752 & 1.4753 & 1.4753 & 1.4752 & 1.4752 \\
RLD & 20000 & 1.4764 & 1.4763 & 1.4763 & 1.4762 & 1.4762 \\
Aj-41rev & 20000 & 1.0002 & 1.0002 & 1.0007 & 1.0012 & 1.0017 \\
Faure & 50000 & 0.9999 & 0.9996 & 0.9991 & 0.9987 & 0.9983 \\
IB & 50000 & 1.4750 & 1.4750 & 1.4750 & 1.4750 & 1.4749 \\
RLD & 50000 & 1.4755 & 1.4755 & 1.4755 & 1.4755 & 1.4755 \\
Aj-41rev & 50000 & 1.0009 & 1.0012 & 1.0019 & 1.0024 & 1.0027 \\
Faure & 70000 & 0.9993 & 0.9989 & 0.9986 & 0.9983 & 0.9980 \\
IB & 70000 & 1.4654 & 1.4654 & 1.4654 & 1.4654 & 1.4653 \\
RLD & 70000 & 1.4710 & 1.4710 & 1.4709 & 1.4709 & 1.4709 \\
Aj-41rev & 70000 & 1.0011 & 1.0013 & 1.0021 & 1.0026 & 1.0029 \\
Faure & 100000 & 0.9997 & 0.9996 & 0.9993 & 0.9990 & 0.9989 \\
IB & 100000 & 1.2785 & 1.2785 & 1.2785 & 1.2785 & 1.2784 \\
RLD & 100000 & 1.3848 & 1.3848 & 1.3848 & 1.3848 & 1.3848 \\
Aj-41rev & 100000 & 1.0013 & 1.0016 & 1.0024 & 1.0028 & 1.0032 \\
\hline & & & & & &
\end{tabular}

crepancy $p$ diagram for our new sequence is at all points below the other sequences. This is a good indication of the high quality of our sequence points.

\section{Numerical Integration}

Another way to compare the quality of sequences is to use them to solve high-dimensional integration problems with numerical methods. Consider the following test integrals:

$$
\begin{aligned}
& I_{1}(f)=\int_{0}^{1} \cdots \int_{0}^{1} \prod_{i=1}^{s} \frac{\pi}{2} \sin \left(\pi x_{i}\right) d x_{1} \cdots d x_{s}=1, \\
& I_{2}(f)=\int_{0}^{1} \cdots \int_{0}^{1} \prod_{i=1}^{s} \frac{\left|4 x_{i}-2\right|+a_{i}}{1+a_{i}} d x_{1} \cdots d x_{s}=1,
\end{aligned}
$$

where the $a_{i}$ are parameters. There are four choices of parameters as follows:

(1) $a_{i}=0$ for $1 \leq i \leq s$

(2) $a_{i}=1$ for $1 \leq i \leq s$ 
TABLE 4: Estimates of $I_{2}(f)$ with $a_{i}=i^{2}$ by using Faure sequences.

\begin{tabular}{lcccccc}
\hline Generator & $N$ & $s=5$ & $s=10$ & $s=20$ & $s=30$ & $s=40$ \\
\hline Faure & 500 & 1.0125 & 1.0115 & 1.0110 & 1.0108 & 1.0106 \\
IB & 500 & 1.5003 & 1.5005 & 1.5006 & 1.5006 & 1.5006 \\
RLD & 500 & 1.5017 & 1.5015 & 1.5015 & 1.5015 & 1.5015 \\
Aj-41rev & 500 & 0.9966 & 0.9936 & 0.9936 & 0.9936 & 0.9936 \\
Faure & 5000 & 0.9988 & 0.9986 & 0.9985 & 0.9984 & 0.9984 \\
IB & 5000 & 1.4838 & 1.4838 & 1.4838 & 1.4838 & 1.4838 \\
RLD & 5000 & 1.4810 & 1.4810 & 1.4810 & 1.4810 & 1.4810 \\
Aj-41rev & 5000 & 0.9993 & 0.9989 & 0.9989 & 0.9989 & 0.9989 \\
Faure & 10000 & 0.9991 & 0.9990 & 0.9989 & 0.9989 & 0.9988 \\
IB & 10000 & 1.4770 & 1.4770 & 1.4770 & 1.4770 & 1.4770 \\
RLD & 10000 & 1.4781 & 1.4781 & 1.4781 & 1.4781 & 1.4781 \\
Aj-41rev & 10000 & 1.0000 & 0.9996 & 0.9996 & 0.9996 & 0.9996 \\
Faure & 20000 & 0.9992 & 0.9991 & 0.9991 & 0.9991 & 0.9991 \\
IB & 20000 & 1.4753 & 1.4753 & 1.4753 & 1.4753 & 1.4753 \\
RLD & 20000 & 1.4764 & 1.4764 & 1.4764 & 1.4764 & 1.4764 \\
Aj-41rev & 20000 & 1.0003 & 1.0002 & 1.0003 & 1.0003 & 1.0003 \\
Faure & 50000 & 1.0000 & 1.0000 & 1.0000 & 1.0000 & 1.0000 \\
IB & 50000 & 1.4750 & 1.4750 & 1.4750 & 1.4750 & 1.4750 \\
RLD & 50000 & 1.4755 & 1.4755 & 1.4755 & 1.4755 & 1.4755 \\
Aj-41rev & 50000 & 1.0006 & 1.0006 & 1.0007 & 1.0007 & 1.0007 \\
Faure & 70000 & 0.9998 & 0.9997 & 0.9997 & 0.9997 & 0.9997 \\
IB & 70000 & 1.4655 & 1.4655 & 1.4655 & 1.4655 & 1.4655 \\
RLD & 70000 & 1.4710 & 1.4710 & 1.4710 & 1.4710 & 1.4710 \\
Aj-41rev & 70000 & 1.0006 & 1.0006 & 1.0007 & 1.0007 & 1.0007 \\
Faure & 100000 & 0.9998 & 0.9998 & 0.9998 & 0.9998 & 0.9998 \\
IB & 100000 & 1.2785 & 1.2785 & 1.2785 & 1.2785 & 1.2785 \\
RLD & 100000 & 1.3848 & 1.3848 & 1.3848 & 1.3848 & 1.3848 \\
Aj-41rev & 100000 & 1.0007 & 1.0007 & 1.0007 & 1.0008 & 1.0008 \\
\hline & & & & & &
\end{tabular}

(3) $a_{i}=i$ for $1 \leq i \leq s$

(4) $a_{i}=i^{2}$ for $1 \leq i \leq s$

Note that the most difficult case is when $a=0$. Because in this case, the importance of all variables is the same and the superposition dimension is approximately the same as the truncation dimension. It is important to know that the larger $a_{i}$, the less important the variables are, and therefore, the effective dimension becomes smaller. The last three choices of the parameters will be considered here [11].

In numerical solution of problems with qMC methods, an accepted procedure is to omit the starting points of the sequence. For example, Fox [12] has suggested that we consider the starting point of the sequence as $n=Q S^{4}-1$. Although, this may lead to better results, note that with this selection, a large amount of points must be omitted. For example, for $s=40$, we have to skip the initial 2825760 points with this formula, which is practically impossible. We found in our research that if we select the $p$ as the starting point, it will significantly improve the results. We will probably get the best result when we start from the $\left(p^{2}+1\right)$ th point.
Therefore, we skip the first 41 points and start $n=42$ in our calculations. For comparison purposes, we present numerical results for original Faure (Faure), our sequence (Aj-41rev), and two types of scrambled Faure sequences, Ibinomial (IB) and random linear digits (RLD).

Now, we compare the numerical results of different scrambled Faure sequences presented in this paper. The estimated values for the test functions are given in Figures 5-8. These figures show that our proposed scramble has a very acceptable convergence compared to other scrambles.

An observation is that estimated values by the matrix $\mathrm{Aj}$ 41rev very close to the actual value. This can be seen in Tables 1-4. The estimation error obtained with this scramble in dimension 40, for the function (16) with parameters $a_{i}=1$, $a_{i}=i$, and $a_{i}=i^{2}$ are at most $11.68 \%, 0.32 \%$, and $0.64 \%$, respectively. However, there are some exceptions. For example, in the first function, when the number of dimensions increases, the accuracy of the estimation decreases. So that, for the dimension 40 , the maximum estimated relative error value is $43.7 \%$.

\section{Conclusion}

We studied the original Faure sequence and some of its recent years introduced scrambles. Then, we introduced a new scrambling matrix based on irrational numbers that its elements are function of square root of base $p$. In Figures 5-8, we have shown that this modified scrambled Faure sequence provides better results than the previous versions of its scrambles. Also, we presented that this modified scrambled Faure sequence has greatly improved the distribution of points. The $2 \mathrm{D}$ designs confirm this claim, good twodimensional projections in successive dimensions. As mentioned in the previous section, using this scramble leads to very small estimated relative errors that can often be ignored. In our next research, we will use deterministic scrambling matrices based on another irrational numbers and primitive roots. The results proved the improvements of accuracy using our new scramble.

\section{Data Availability}

The data is contained in the article itself.

\section{Conflicts of Interest}

The authors declares that there is no conflict of interest regarding the publication of this paper.

\section{References}

[1] A. B. Owen, "Monte Carlo, quasi-Monte Carlo, and randomized quasi-Monte Carlo," in Monte Carlo and Quasi-Monte Carlo Methods, H. Niederreiter and J. Spanier, Eds., pp. 8697, Springer-Verlag, Berlin Heidelberg, 1998.

[2] S. Tezuka, "A generalization of Faure sequences and its efficient implementation,” Technical Report RT0105, IBM. Tokyo Research Laboratory, 1994. 
[3] B. Vandewoestyne, H. Chi, and R. Cools, "Computational investigations of scrambled Faure sequences," Mathematics and Computers in Simulation, vol. 81, pp. 522-535, 2003.

[4] H. Faure, "Variations on (0, s)-sequences," Journal of Complexity, vol. 17, no. 4, pp. 741-753, 2015.

[5] J. Matou'sek's, "On the L2-discrepancy for anchored boxes," Journal of Complexity, vol. 14, pp. 527-556, 1998.

[6] S. Tezuka and H. Faure, "I-binomial scrambling of digital nets and sequences," Journal of Complexity, vol. 19, no. 6, pp. 744757, 2003.

[7] P. Bratley, B. L. Fox, and H. Niederreiter, "Implementation and tests of low-discrepancy sequences," ACM Transactions on Modeling and Computer Simulation, vol. 2, no. 3, pp. 195-213, 1992.

[8] H. Chi, Scrambled Quasirandom Sequences and their Applications, [Ph.D. thesis], Florida State University, Tallahassee, FL, USA, 2004.

[9] B. Fathi Vajargah and A. Eskandari Chechaglou, "New modified scrambled faure sequences," Communications in Statistics - Simulation and Computation, vol. 44, no. 3, pp. 666-682, 2012.

[10] B. Vandewoestyne, Quasi-Monte Carlo Techniques for the Approximation of High-Dimensional Integrals, [Ph.D. theses], Advisor Ronald Cools, 2008.

[11] X. Wang and K. T. Fang, "The effective dimension and quasiMonte Carlo integration," Journal of Complexity, vol. 19, no. 2, pp. 101-124, 2003.

[12] B. L. Fox, "Algorithm 647: implementation and relative efficiency of quasirandom sequence generators," ACM Transactions on Mathematical Software, vol. 12, no. 4, pp. 362-376, 1986. 